\title{
Aplicação da Função de Estabilidade Mestra em um Modelo de Redes de Economias Acopladas
}

\author{
Amaury S. Amaral ${ }^{1}$ \\ Departamento de Contabilidade \\ Pontifícia Universidade Católica de São Paulo, São Paulo, SP \\ Victor E. Camargo M. ${ }^{2}$ \\ Núcleo Interdisciplinar de Sistemas Complexos, USP, São Paulo, SP \\ Fernando F. Ferreira ${ }^{3}$ \\ Núcleo Interdisciplinar de Sistemas Complexos, USP, São Paulo, SP
}

\begin{abstract}
Resumo. Neste trabalho estudamos a interação entre sistemas dinâmicos não linear que representam a macroeconomia simplificada. O modelo é uma versão modificada e estendida do oscilador de Van der Pol. Considerou-se apenas três variáveis de estados: a entrada de capital estrangeiro, a poupança das famílias e o produto interno bruto. Com efeito, analisamos possíveis padrões que resultam desta dinâmica bem como mudanças estruturais quando se varia o fluxo de capital estrangeiro, escolhido aqui como o parâmetro de controle. Há uma riqueza de comportamentos dinâmicos que podem ser obtidos via simulações numéricas. Para certos valores do parâmetro de controle ocorre a sincronização entre as economias. Contudo, elas podem ser instáveis. Assim, aplicamos o método da função de estabilidade mestra (Master Stability Function) para avaliar a estabilidade. O resultado principal foi observar a ocorrência de sincronizações instáveis quando o acoplamento foi unidirecional, e estável no caso bidirecional. Além disso, o método de análise permite que se explore um número arbitrário de economias acopladas com diferentes topologias de redes.
\end{abstract}

Palavras-chave. Rede complexa, Economia de rede, Sincronização, Estabilidade, Oscilador de Van der Pol.

\section{Introdução}

A economia afeta a vida de bilhões de pessoas que, por falhas de mercado, sofrem devido ao desemprego, crescimento do produto interno bruto baixo, concentração de renda e pobreza. Um dos grandes problemas é a dificuldade de prever e previnir as crises. De modo geral, a previsibilidade em sistemas econômicos representa um desafio para diversos campos de estudo. Estes sistemas são formados por um elevado número de agentes heterogêneos que interagem de forma não linear no nível microscópico, que reagem às informações de indicadores macroecônomicos (feedbacks) e ainda sofrem influências de outras

\footnotetext{
${ }^{1}$ asamaral@pucsp.br

${ }^{2}$ victor.camargo11@usp.br

${ }^{3}$ ferfff@gmail.com
} 
economias pelo fluxo de capital entre elas. A quantidade de parâmetros envolvidos, a não linearidade e a dificuldade de capturar via modelagem as expectativas dos agentes estão na origem do problema. Por outro lado, nossa incapacidade presente de produzir bons modelos deixam muitas perguntas em aberto. Não somos capazes de evitar crises econômicas e controlar o risco sistêmico causado pela propagação dos efeitos de uma recessão ou crash de um país sobre seus parceiros. Assim, pode-se dizer que é importante estudar modelos simplificados para entender os mecanismos fundamentais dos padrões coletivos de economias acopladas. Os sistemas econômicos são exemplos de sistemas complexos e sua imprevisibilidade deve-se a não linearidade, à semelhança do que acontece nos sistemas caóticos. Como é sabido, ainda existe controvérsia sobre a presença de caos em dados econômicos devido ao tamanho das séries empíricas e à suposta alta dimensionalidade do sistema [10]. Entretanto, as economias apresentam comportamentos coletivos como mudanças estruturais (bifurcações) que podem ser estudados por meio da modelagem matemática $[1,15]$. O aspecto interessante é que os padrões observados em sistemas ecológicos ou físicos também aparecem em outros sistemas tais como econômicos e fisiológicos. Por exemplo, oscilações e sincronizações de fase são comportamentos dinâmicos estudados na Física, cujos padrões também aparecem em populações de vaga-lumes, em séries de batimentos cardíacos ou nos ciclos circadianos em humanos [12]. Assim, não é surpresa que os modelos paradigmáticos para o estudo das oscilações na Física possam ser adaptados para estudar ciclos econômicos.

Algumas variáveis econômicas agregadas exibem um tipo de flutuação chamada de ciclos de negócios. Estes ciclos consistem em expansões que ocorrem quase ao mesmo tempo em muitas atividades econômicas, seguidas de recessões, contrações e recuperação [4]. Essa sequência de mudanças é recorrente, mas não apresenta duração e períodos bem comportados. Entre as variáveis, podemos citar consumo, investimento, produto interno bruto e estoque de capital, que seguem um movimento oscilatório incorporando um vasto espectro de ciclos. Esses comportamentos oscilatórios tem causa endógena e podem ser descritos por meio de equações diferenciais não lineares $[5,15]$. Vale ressaltar que estas economias podem ser abertas e realizar trocas com outras economias sujeitas a flutuações. Como as economias interagentes podem estar em fases diferentes dos ciclos de negócios, é interessante estudar o que acontece com várias economias acopladas. Considerando cada economia como um sistemas dinâmico complexo, pode-se usar inúmeras técnicas matemáticas para caracterizar os padrões que elas exibem ao variarmos os parâmetros relevantes, bem como estudar a estabilidade das soluções. O estudo da estabilidade se dá por meio de técnicas mais avançadas, como por exemplo a função de estabilidade mestra, que será apresentada na seção de metodologia [7,12,13]. No presente trabalho examinamos o compartamento dinâmico entre três economias que podem ser idênticas ou não, que foram acopladas por meio da variável troca de capital. Estas economias foram aproximadas por um modelo de oscilador de ciclo-limite. O modelo será descrito na próxima seção, contudo podemos dizer que ele é uma modificação do sistema bidimensional de Van der Pol $[3,14]$ com a adição de um termo de feedback que representa as trocas internacionais. Além disso, do ponto de vista econômico, o sistema simula a fuga de capitais observada nos países menos desenvolvidos. Então, neste trabalho é examinado o efeito da entrada e saída de capital entre dois sistemas econômicos conjugados. 


\section{Modelo Macroeconômico}

Bouali et al. [2] propuseram uma modificação do oscilador de Van de Pol bidimensional que fosse capaz de representar um sistema macroecônomico idealizado. Para isto, eles introduziram uma terceira equação e fizeram a interpretação das variáveis de estado no contexto macroeconômico. O modelo é descrito por três equações diferenciais ordinárias, a saber:

$$
\left\{\begin{array}{l}
\dot{x}=m \cdot y+p \cdot x \cdot\left(d-y^{2}\right), \\
\dot{y}=-x+c \cdot z \\
\dot{z}=s \cdot x-r \cdot y
\end{array}\right.
$$

As variáveis de estado $x, y$ e $z$ representam a poupança das famílias, o produto interno bruto (PIB) e a entrada de capital estrangeiro respectivamente. Os parâmetros $m, p$, $d, c, s$ e $r$ são positivos e representam respectivamente os índices: propensão marginal a consumir, fator de lucro capitalizado do investimento, valor do PIB, fator de saida do capital, fator de propensão a poupar e o fator do endividamento.

Este sistema possui três pontos de equilíbrio: $E_{1}=(0,0,0), E_{2}=(\alpha, s \alpha / r,-\alpha / c)$ e $E_{3}=-E_{2} \operatorname{com} \alpha=[p d+m(s / r)] /\left[(s / r)^{2} p\right]$ [3]. O Jacobiano do sistema (eq.1) é $|J(x, y, z)|=c s(m+2 p x y)+\operatorname{crp}\left(d-y^{2}\right)$. Para os parâmetros $(m, p, d, c, s, r)=$ $(0.02,0.4,1.0,50,10,0.1)$ o sistema exibe comportamento caótico conforme apresentado por Bouali utilizando-se do algoritmo de Wolf et al. [16], ilustrado na figura 1.

O sistema descrito pelas equações 1 exibem uma riqueza de comportamentos dinâmicos quando se varia o parâmetro de controle $c$ conforme bem discutido por Bouali [3]. Aqui iremos apenas mostrar a reconstrução do atrator 3D e o expoente de Lyapunov como função do parâmetro de controle $c$ conforme figura 1.
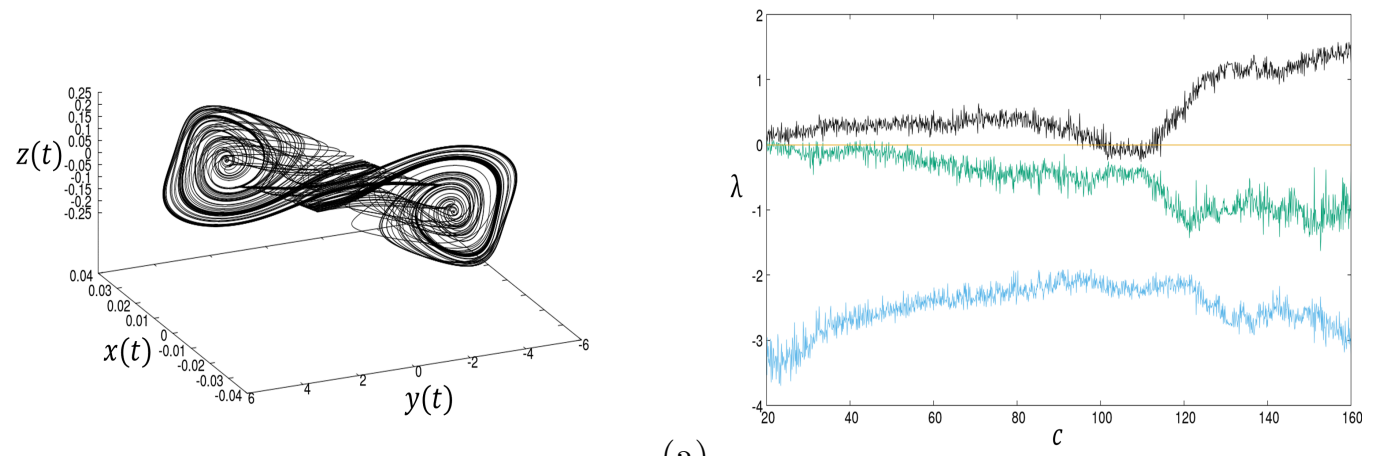

(a)

Figura 1: (a) Atrator tridimensional do sistema (eq 1). (b) Expoentes de Lyapunov $\lambda$ como função do parâmetro de controle $c$. As figuras foram gerada com os seguintes parâmetros: $(m, p, d, c, s, r)=(0.02,0.4,1.0,50,10,0.1)$ respectivamente. 


\subsection{Sistema Macroeconômico Acoplado}

Recentemente, Volos et al. [15] estudaram a interação de duas economias. Para isto, eles acoplaram dois modelos de Bouali na variável que descreve o fluxo de entrada de capital do exterior. A generalização para $N$ economias, seguindo tal modelo de acoplamento difusivo proposto, é dado pelo seguinte conjunto de equações diferencias:

$$
\left\{\begin{array}{l}
\dot{x}_{i}=m y_{i}+p x_{i}\left(d-y_{i}^{2}\right), \\
\dot{y}_{i}=-x_{i}+c z_{i}, \\
\dot{z}_{i}=s x_{i}-r y_{i}+\xi_{i}\left(z_{i+1}-z_{i}\right),
\end{array}\right.
$$

com $i=1,2, \ldots, N$. A variação $z_{i+1}-z_{i}$ representa o saldo na balança comercial do país $i$ com o país $i+1$. A balança comercial pode ser utilizada para financiar os déficits orçamentários do governo ou pode ser usado para aumentar a produção. Assim, $\xi_{i}$ mede o efeito da balança comercial sobre a economia. Na próxima seção, vamos estudar a estabilidade deste sistema dinâmico.

\section{Análise da Função de Estabilidade Mestra}

A evolução temporal dos estados de um sistema dinâmico determinístico isolado é determinada por $m$ equações:

$$
\dot{\mathbf{x}}=\mathbf{F}(\mathbf{x}),
$$

aqui o vetor $\mathbf{x}$ descreve o estado de um oscilador e $\mathbf{F}(\mathbf{x})$ define o vetor do campo de velocidade do sistema. É possível formar uma rede a partir do acoplamento de $N$ subsistemas isolados:

$$
\dot{\mathbf{x}}_{i}=\mathbf{F}\left(\mathbf{x}_{i}\right)-\varepsilon \sum_{j=1}^{N} G_{i j} \mathbf{H}\left(\mathbf{x}_{j}\right),
$$

com $i=1,2, \ldots, N, \mathbf{H}\left(\mathbf{x}_{j}\right)$ é a saída do j-ésimo nó, $\mathbf{G}$ é matriz de acoplamento determinada pela topologia da rede e $\varepsilon$ é o parâmetro global de acoplamento. A matriz $\mathbf{G}=G_{i j}$ satisfaz a condição de soma zero das linhas $\sum_{j=1}^{N} G_{i j}=0, \forall i$. O estado de sincronização corresponde a $\mathbf{x}_{i}=\mathbf{x}_{s}, \forall i$ com $\dot{\mathbf{x}}_{s}=\mathbf{F}\left(\mathbf{x}_{s}\right)$ que é uma solução exata da (eq.4).

Para analisar a estabilidade do estado sincronizado, nós perturbamos o estado de cada nó na rede sobre o estado sincronizado $\mathbf{x}_{i}(t)=\mathbf{x}_{s}(t)+\delta \mathbf{x}_{i}(t)$, e expandimos a (eq.4) em uma série de Taylor de 1a. ordem, então os desvios $\delta \mathbf{x}_{i}(t)=\mathbf{x}_{i}(t)-\mathbf{x}_{s}(t)$ satisfazem as equações variacionais:

$$
\delta \dot{\mathbf{x}}_{i}=\mathbf{D F}\left(\mathbf{x}_{s}\right) \delta \mathbf{x}_{i}-\varepsilon \sum_{j=1}^{N} G_{i j} \mathbf{D H}\left(\mathbf{x}_{s}\right) \delta \mathbf{x}_{j},
$$


sendo $\mathbf{D F}\left(\mathbf{x}_{s}\right)$ e $\mathbf{D H}\left(\mathbf{x}_{s}\right)$ matrizes jacobianas $m \times m$ correspondentes às funções vetoriais avaliadas em $\mathbf{x}_{s}(t)$.

Seguindo a ideia proposta por Pecora and Carroll [13], a (eq.5) pode ser diagonalizada em $N$ modos próprios desacoplados na forma de bloco

$$
\dot{\mathbf{w}}_{i}=\left[\mathbf{D F}\left(\mathbf{x}_{s}\right)-\varepsilon \mu_{i} \mathbf{D H}\left(\mathbf{x}_{s}\right)\right] \mathbf{w}_{i},
$$

sendo que $\mathbf{w}_{i}$ representa diferentes modos de perturbações de um estado sincronizado e os $\mu_{i}$ são os autovalores de $G$, com $i=1,2, \ldots, N$, os quais podem serem ordenados como $\mu_{1}=0 \leq \operatorname{Re}\left(\mu_{2}\right) \leq \ldots \leq \operatorname{Re}\left(\mu_{N}\right)$.

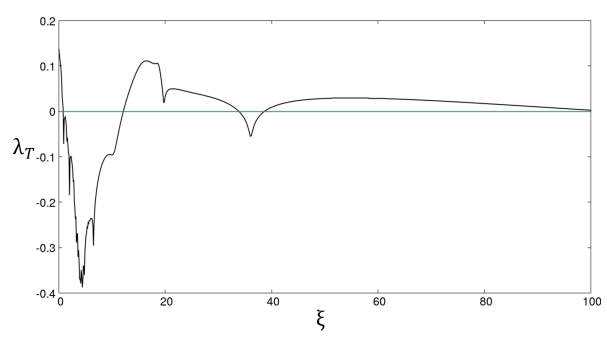

(a)
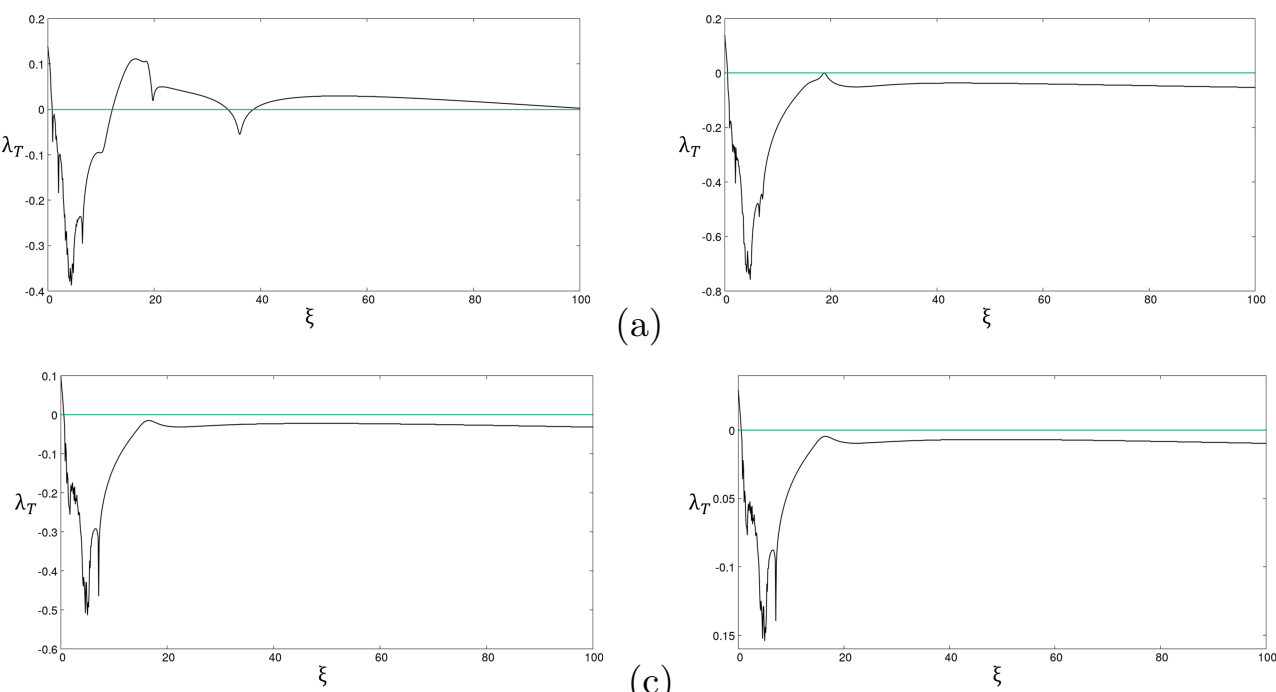

(c)

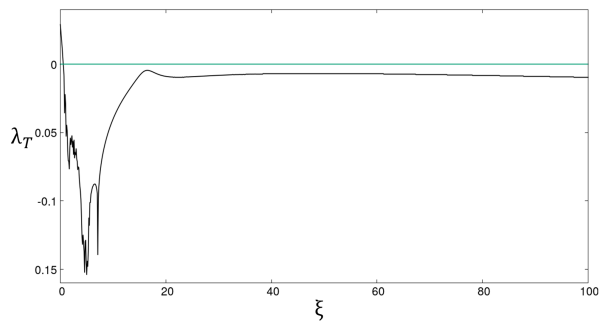

(d)

Figura 2: Função de estabilidade mestra $\lambda_{T}$ para $(a)$ acoplamento unidirecional $\xi=0$ para $\dot{z}_{1}$, (b) acoplamento bidirecional tanto em $\dot{z}_{1}$ como em $\dot{z}_{2},(c)$ para uma rede de $N=3$ e (d) para $N=10$

\section{Resultado e Discussão}

As economias (eq.2) foram acoplada por meio dos parâmetros de controle $\xi_{i}$. Preliminarmente, consideramos o caso em que há apenas duas economias, sendo uma a 'mestre' $\xi_{1}=0$ e a outra $\xi_{2}=\xi>0$ a 'escrava', também chamado de modelo unidirecional [15]. Neste caso, a economia 1 exerce influência sobre a economia 2, mas não o inverso. Por meio da análise da função de estabilidade mestra $\lambda_{T}$ pudemos estudar a estabilidade da sincronização deste tipo de acoplamento, reportado na figura 2(a). Inicialmente, observamos que não ocorre sincronização para baixos valores de $\xi$, uma vez que os parâmetros escolhidos colocam os subsistemas na fase caótica e ainda a força do acoplamento não é capaz de promover a sincronzação. O aumento no valor do parâmetro de controle leva à sincronização estável do sistema. No entanto, com o aumento do valor de $\xi>15$ o sistema desestabiliza, retornando a estabilidade no intervalo de $\xi=\left(\begin{array}{ll}35 & \text { a } 38\end{array}\right)$. Para valores superiores, no intervalo estudado de $\xi=(39$ a 100) o sistema perde a estabilidade. No caso 
bidirecional, estudado para $N=2,3$ e 10, o sistema entra na fase estável e não se observou a volta para o regime instável (ver figura 2). Apesar de que cada economia (subsistema) ser intrinsecamente caótica, o acoplamento difusivo do modelo permite a emergência da sincronização de forma estável.

O modelo macroeconômico aqui descreve a relação entre poupança, PIB e entrada de capital estrangeiro. Ele é bem rudimentar, e possui premissas que podem ser questionadas pelos economistas e possivelmente melhoradas. A equação que descreve a variação da poupança depende da variação do PIB, e concomitantemente a poupança cresce com o aumento do PIB, enquanto a condição $b-y^{2}>0$ for satisfeita. Caso contrário, o nível de poupança diminui, o que pode significar um aumento no consumo e uma pressão para o incremento da inflação. O PIB também é afetado pelo nível de poupança e pela entrada de capital externo. Contudo, isso leva à possibilidade de uma influência do exterior sobre a economia nacional via fluxo de entrada de capital $(z)$. Notamos que as economias podem atuar de forma cooperativa e ter como resultado coletivo a sincronização entre as variáveis macroeconômicas, simplesmente pelo ajuste do parâmetro de controle. O comportamento oscilatório das variáveis macroeconômicas emula os ciclos econômicos, mais ainda, as flutuações são endógenas, emergentes, podendo ser auto-sustentáveis ou caóticas. Neste modelo, os períodos de oscilação dependem do valor de $\xi_{i}$. Em dados empíricos, esses períodos podem variar de poucos anos até decadas. Assim, ao introduzir ruídos no modelo permitirá reproduzir cenários mais realísticos. Porém, também vai demandar o uso de alguns métodos, entre eles o da função de estabilidade mestra que permitira tirar conclusões mais realistas. Nosso foco não foi apresentar um modelo macroeconômico realista, mas sim, operar uma técnica de análise da estabilidade da sincronização entre economias acopladas. Avançamos em relação ao trabalho de Volos et al. [15] que discutiram o caso de apenas duas economias acopladas. Muito embora eles tenham demostrado que este sistema (de duas economias) podem sincronizar, faltou discutir se os estados sincronizados são estáveis. A fim de estender a análise para uma rede de economias com um número arbitrário de paises $N$ optamos por usar a função de estabilidade mestra.

Neste trabalho apresentamos a versão generalizada do modelo de Bouali [3,15] em uma rede acoplada. Verificamos que este modelo encapsula uma riqueza de comportamentos dinâmicos complexos que permite estudar vários regimes tais como os ciclos econômicos ou cenários aperiódicos e instáveis. Uma versão simplificada como a apresentada aqui é importante para endereçar questões específicas que considere efeitos globais e influências mútuas, simétricas e assimétricas de economias acopladas em redes. A contribuição deste trabalho foi aplicar o método da função de estabilidade mestra para o estudo de uma rede de sistemas que representa qualitativamente uma macroeconomia. Como sugestão de trabalhos futuros podemos estudar topologias de redes mais realistas, fazer um estudo mais detalhado do efeito da variação dos parâmetros de modelo generalizado e ainda confrontar com dados empíricos. De forma geral, o método de análise pode ser aplicado para o estudo de modelos dinâmicos não lineares mais sofisticados.

\section{Agradecimentos}

Victor Camargo e FFF agradecem à CAPES pelo suporte financeiro e Amaury S. Amaral agradece à PUCSP, em especial à SBMAC e à Fundação Instituto de Física Teórica. 


\section{Referências}

[1] Barnett, W. and Duzhak, E. Empirical assessment of bifurcation regions within New Keynesian models. Economic Theory. 45, 99-128 (2010).

[2] Bouali, S., Buscarino, A., Fortuna, L., Frasca, M. and Gambuzza, L. Emulating complex business cycles by using an electronic analogue. Nonlinear Analysis: Real World Applications. 13, 2459-2465 (2012).

[3] Bouali, S. Feedback Loop in Extended Van Der Pol's Equation Applied to an Economic Model of Cycles. International Journal Of Bifurcation And Chaos. 9, 745-756 (1999).

[4] Calderón, C. and Fuentes, J. Have business cycles changed over the last two decades? An empirical investigation. Journal Of Development Economics. 109 pp. 98-123 (2014).

[5] Goodwin, R. A growth cycle, in capitalism and growth, C. Feinstein, ed. Cambridge University Pres, (1967).

[6] Hramov, A. E., Khramova, A. E., Koronovskii, A. A., and Boccaletti, S. Synchronization in networks of slightly nonidentical elements. International Journal of Bifurcation and Chaos, v. 18, n. 03, p. 845-850, (2008).

[7] Huang, L., Chen, Q., Lai, Y. and Pecora, L. Generic behavior of master-stability functions in coupled nonlinear dynamical systems. Physical Review E.80, 036204 (2009).

[8] J. Doyne Farmer, Economics Needs to Treat the Economy as a Complex System. Conferência "Rethinking Economics and Politics". Oxford: INET - Institute for New Economic Thinking, pp. 1-15, (2012).

[9] Korotayev, A. Tsirel, S. A spectral analysis of world GDP dynamics: Kondratieff waves, Kuznets swings, Juglar and Kitchin cycles in global economic development, and the 2008-2009 economic crisis. Structure And Dynamics. 4, (2010).

[10] Kyrtsou, C. and Vorlow, C. Complex dynamics in macroeconomics: A novel approach. Springe, (2005).

[11] N. F. Johnson, Simply Complexity: A Clear Guide to Complexity Theory. Oxford: Oneworld, (2007).

[12] Monteiro Henrique, L. Sistemas Dinâmicos. São Paulo. Livraria da Física, (2014).

[13] Pecora, L. and Carroll, T. Master stability functions for synchronized coupled systems. Physical Review Letters. 80, 2109 (1998).

[14] Van der Pol, B. On "relaxation-oscillations". The London, Edinburgh, And Dublin Philosophical Magazine And Journal Of Science. 2, 978-992 (1927).

[15] Volos, C., Kyprianidis, I. and Stouboulos, I. Synchronization phenomena in coupled nonlinear systems applied in economic cycles. Wseas Trans. Syst. 11, 681-690 (2012).

[16] Wolf, A., Swift, J., Swinney, H. and Vastano, J. Determining Lyapunov exponents from a time series. Physica D: Nonlinear Phenomena.16, 285-317 (1985). 